\title{
Comparing the information capacity of Laguerre-Gaussian and Hermite-Gaussian modal sets in a finite-aperture system
}

\author{
Sara Restuccia, ${ }^{1,}{ }^{*}$ Daniel Giovannini, ${ }^{2}$ Graham Gibson, ${ }^{1}$ And \\ MILES PADGETT ${ }^{1}$ \\ ${ }^{1}$ SUPA, School of Physics and Astronomy, University of Glasgow, Glasgow G12 8QQ, UK \\ ${ }^{2}$ The Edward S. Rogers Department of Electrical and Computer Engineering, University of Toronto, \\ Ontario M5S 3G4, Canada \\ s.restuccia.1@research.gla.ac.uk
}

\begin{abstract}
Using a spontaneous parametric down-conversion process to create entangled spatial states, we compare the information capacity associated with measurements in the HermiteGaussian and Laguerre-Gaussian modal basis in an optical system of finite aperture. We show that the cross-talk imposed by the aperture restriction degrades the information capacity. However, the Laguerre-Gaussian mode measurements show greater resilience to cross talk than the HermiteGaussian, suggesting that the Laguerre-Gaussian modal set may still offer real-world advantages over other modal sets.
\end{abstract}

\section{(c) 2016 Optical Society of America}

OCIS codes: (270.5585) Quantum information and processing; (270.5568) Quantum cryptography.

\section{References and links}

1. A. E. Willner, H. Huang, Y. Yan, Y. Ren, N. Ahmed, G. Xie, C. Bao, L. Li, Y. Cao, Z. Zhao, J. Wang, M. P. J. Lavery, M. Tur, S. Ramachandran, A. F. Molisch, N. Ashrafi, and S. Ashrafi, "Optical communications using orbital angular momentum beams," Adv. Opt. Photon. 7, 66-106 (2015).

2. M. Krenn, R. Fickler, M. Fink, J. Handsteiner, M. Malik, T. Scheidl, R. Ursin, and A. Zeilinger, "Communication with spatially modulated light through turbulent air across Vienna," New J. Phys. 16, 113028 (2014).

3. A. E. Willner, J. Wang, and H. Huang, "A different angle on light communications," Science 337, 655-656 (2012).

4. J. Leach, B. Jack, J. Romero, A. K. Jha, A. M. Yao, S. Franke-Arnold, D. G. Ireland, R. W. Boyd, S. M. Barnett, and M. J. Padgett, "Quantum correlations in optical angle-orbital angular momentum variables," Science 329, 662-665 (2010).

5. L. Allen, M. W. Beijersbergen, R. J. C. Spreeuw, and J. P. Woerdman, "Orbital angular-momentum of light and the transformation of Laguerre-Gaussian laser modes," Phys. Rev. A 45, 8185-8189 (1992).

6. A. M. Yao and M. J. Padgett, "Orbital angular momentum: origins, behavior and applications," Adv. Opt. Photon. 3, $161(2011)$.

7. G. Gibson, J. Courtial, M. J. Padgett, M. Vasnetsov, V. Pas'ko, S. M. Barnett, and S. Franke-Arnold, "Free-space information transfer using light beams carrying orbital angular momentum," Opt. Express 12, 5448-5456 (2004).

8. J. Wang, J.-Y. Yang, I. M. Fazal, N. Ahmed, Y. Yan, H. Huang, Y. Ren, Y. Yue, S. Dolinar, M. Tur, and A. E. Willner, "Terabit free-space data transmission employing orbital angular momentum multiplexing," Nat Photonics 6, 488-496 (2012).

9. A. Mair, A. Vaziri, G. Weihs, and A. Zeilinger, "Entanglement of the orbital angular momentum states of photons," Nature 412, 313-316 (2001).

10. M. W. Beijersbergen, L. Allen, H. E. L. O. van der Veen, and J. P. Woerdman, "Astigmatic laser mode converters and transfer of orbital angular momentum," Opt. Commun. 96, 123-132 (1993).

11. N. Zhao, X. Li, G. Li, and J. M. Kahn, "Capacity limits of spatially multiplexed free-space communication," Nat. Photonics 8(12), 822-826 (2015).

12. R. L. Phillips and L. C. Andrews, "Spot size and divergence for Laguerre-Gaussian beams of any order," Appl. Opt. 22, 643-644 (1983).

13. M. V. Berry, "Faster than Fourier," in Quantum Coherence and Reality, J. S. Anandan and J. L. Safko, eds. (World Scientific, 1994), pp 55-65.

14. P. J. S. G. Ferreira and A. Kempf, "Superoscillations: faster than the nyquist rate," IEEE Trans. Signal Process. 54, 3732-3740

15. V. Y. Bazhenov, M. V. Vasnetsov, and M. S. Soskin, "Laser-beams with screw dislocations in their wave-fronts," JETP Lett. 52, 429-431 (1990).

16. J. Arlt, K. Dholakia, L. Allen, and M. J. Padgett, "The production of multiringed Laguerre-Gaussian modes by computer-generated holograms,” J. Mod. Opt. 45, 1231-1237 (1998). 
17. J. E. Curtis, B. A. Koss, and D. G. Grier, "Dynamic holographic optical tweezers," Opt. Commun. 207, 169-175 (2002).

18. E. Yao, S. Franke-Arnold, J. Courtial, M. J. Padgett, and S. M. Barnett, "Observation of quantum entanglement using spatial light modulators," Opt. Express 14, 1308-13094 (2006).

19. T. W. Clark, R. F. Offer, S. Franke-Arnold, A. S. Arnold and N. Radwell, "Comparison of beam generation techniques using a phase only spatial light modulator," Opt. Express 24, 6249-6264 (2016).

20. R. W. Boyd, B. Rodenburg, M. Mirhosseini, and S. M. Barnett, "Influence of atmospheric turbulence on the propagation of quantum states of light using plane-wave encoding," Opt. Express 19, 18310-18317 (2011).

21. V. D’Ambrosio, E. Nagali, S. P. Walborn, L. Aolita, S. Slussarenko, L. Marrucci, and F. Sciarrino, "Complete experimental toolbox for alignment-free quantum communication," Nat. Commun. 3, 961 (2012).

22. C. E. Shannon, "A mathematical theory of communication," Bell Syst. Tech. J. 27, 379-423 (1948).

23. V. D. Salakhutdinov, E. R. Eliel, and W. Löffler, "Full-field quantum correlations of spatially entangled photons," Phys. Rev. Lett. 108, 173604 (2012).

24. F. M. Miatto, D. Giovannini, J. Romero, S. Franke-Arnold, S. M. Barnett, and M. J. Padgett, "Bounds and optimisation of orbital angular momentum bandwidths within parametric down-conversion systems," Eur. Phys. J. D 66, 20736 (2012).

25. S. S. R. Oemrawsingh, X. Ma, D. Voigt, A. Aiello, E. R. Eliel, G. W. 't Hooft, and J. P. Woerdman, "Experimental demonstration of fractional orbital angular momentum entanglement of two photons," Phys. Rev. Lett. 95, 240501 (2005).

26. M. McLaren, M. Agnew, J. Leach, F. S. Roux, M. J. Padgett, R. W. Boyd, and A. Forbes, "Entangled Bessel-Gaussian beams," Opt. Express 20, 23589-23597 (2012).

27. R. Fickler, R. Lapkiewicz, W. N. Plick, M. Krenn, C. Schaeff, S. Ramelow, and A. Zeilinger, "Quantum entanglement of high angular momenta," Science 338, 640-643 (2012).

28. J. Leach, B. Jack, J. Romero, M. Ritsch-Marte, R.W. Boyd, A.K. Jha, S.M. Barnett, S. Franke-Arnold, and M. J. Padgett, "Violation of a Bell inequality in two-dimensional orbital angular momentum state-spaces," Opt. Express 17, 8287-8293 (2009).

29. B. Jack, A. M. Yao, J. Leach, J. Romero, S. Franke-Arnold, D. G. Ireland, S. M. Barnett, and M. J. Padgett, "Entanglement of arbitrary superpositions of modes within two-dimensional orbital angular momentum state spaces," Phys. Rev. A 81, 043844 (2010).

30. S. Restuccia, "Comparing the information capacity of Laguerre-Gaussian and Hermite-Gaussian modal sets in a finite-aperture system," http://dx.doi.org/10.5525/gla.researchdata.361 (2016).

\section{Introduction}

There is currently interest in the use of spatial optical modes for encoding both quantum and classical information [1-3]. At the most fundamental level, a photon has many degrees of freedom: polarisation, frequency, position, spatial mode, etc., any one of which can be used to encode information; all create additional channels. Within the quantum context, some of these degrees of freedom are incompatible. For example, the extent to which one can encode information simultaneously and independently on both the position and momentum variables is limited by the uncertainty principle. The same limitation is true for angular position and angular momentum [4].

Angular momentum is usually associated with the photon spin, but as recognised in 1992 [5], the orbital angular momentum (OAM) is associated with the spatial optical mode. Many groups around the world have considered both the fundamental properties and possible applications of OAM [6]. In particular, one area where OAM seems to provide new opportunities is in optical communication. Following a simple demonstration of OAM multiplexing in 2004 [7], many groups have pursued this line of study [3], reaching terabit data rates [8]. Investigations using these angular momentum-carrying modes have also led to new tests of quantum mechanics in high-dimensional state spaces [9] and angular test of the EPR paradox [4] . Beyond these explorations of quantum mechanics has been the use of Laguerre-Gaussian modes for encoding information in the photon s spatial degree of freedom [7], thereby increasing the amount of information that can be carried by a single photon [8] . The Laguerre-Gaussian (LG) modal set is a discrete cylindrical basis, and just one of the orthogonal bases in which any arbitrary light beam can be represented. What sets the LG modal set apart from other modal sets is an azimuthal phase-term, given by $\exp (-i l \theta)$, that describes a helical phasefront and hence the OAM corresponding to $l \hbar$ per photon [5]. These modes also have a radial mode index, $p$, giving 
the number of concentric, high-intensity, rings in the transverse cross-section. The LG mode described by the indices $l, p$ can be compared to the more familiar Hermite-Gaussian modes (HG) described by the indices $m, n$ that are frequently observed from misaligned laser cavities.

Interestingly, the HG and LG modes are related to each other in that a suitably specified pair of cylindrical lenses transform any HG mode of indices $m, n$ into a LG mode of indices $l, p$, where $l=m-n$ and $p=\min (m, n)$ [10]. For both modal sets of order $N=m+n=|l|+2 p$ there are $N+1$ orthogonal modes (table 1 ).

Table 1. Hermite-Gaussian and Laguerre-Gausian mode indices and intensity profiles for mode order $N \leq 3$. The arrow on the LG modal cross-section indicates the sense of the azimuthal phase term.

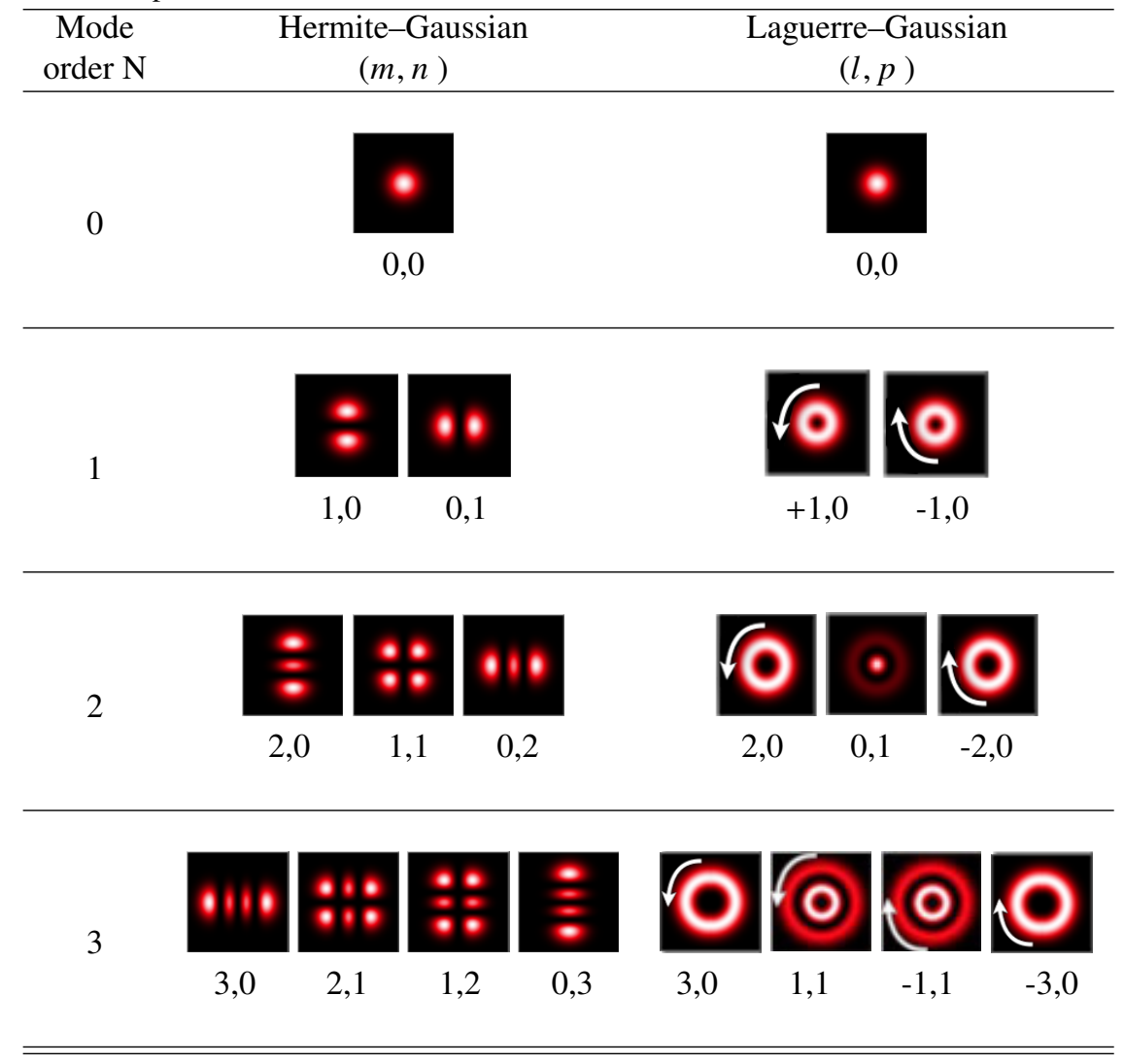

When applied to the encoding of information, it might be expected that all modal sets offer the same information capacity, where the number of modes that can be transmitted with low loss is comparable to the Fresnel number of the optical system [11]. Put simply, for beams of a given radius, modes of higher order diverge more quickly [12], requiring a collection telescope of larger aperture. The aperture required scales with the number of orthogonal modes one wishes to measure. If arbitrarily high levels of loss can be tolerated then this number of orthogonal modes is, in principle, unbounded: see for example literature on superoscillations $[13,14]$.

Over the years, there have been many method reported for both the generation and transformation of optical spatial modes. However, for generation and measurement at the single-photon level, the most common method to generate and measure these modes is the use of diffractive optical elements to imprint the required phase onto a Gaussian beam, creating an approximation to the desired mode in the far-field [15]. This technique has also been extended to create modes 
with $p>0$ by the inclusion of a radial discontinuity into the diffractive pattern [16]. Rather than using elements of a fixed design, it is now commonplace to implement these diffractive elements using programmable spatial light modulators (SLM), thereby allowing practical switching between different states [17].

In addition to generation, the same SLM approach can be used for detection, where an incident mode is phase-flattened by the spatial light modulator such that it can be coupled into the fundamental mode of a single-mode fibre [18]. Unlike the cylindrical lenses discussed above, a phase-only spatial light modulator, when illuminated with a Gaussian mode, creates only approximations of the desired mode. This is particularly evident in LG modes in that the orthogonality of modes requires amplitude-sensitive detection. However, in using this phase-only approach, what is lost in modal fidelity is offset by the ease of use and generality of the conversion process. More sophisticated designs of diffractive element can produce purer modes, but only at the expense of reduced conversion efficiency [19].

The equivalence in the Fresnel number capacity limit for all spatial modes based upon their mode order does not mean that all systems are equal. From a technological perspective some spatial modes are easier to generate or detect than others: certain spatial modes might correspond to the eigenmodes of a particular transmission system, and hence exhibit reduced cross talk, or be robust to specific aberrations [20] or symmetry shifts [21]. But in general, for a fixed Fresnel number, and therefore a limiting channel capacity, there is superficially no reason to favour one modal set over another. However, the experimental comparison between LG and HG modes is more complicated. The trade-off between the measured orthogonality between modes, transmission loss and size of a truncating aperture is complicated. The present work seeks to compare the information capacity of a LG and HG modal basis in the measurement of the quantum correlations between spatial modes.

\section{Method and results}

For the purpose of this study, there are two length scales of importance. Firstly, the lateral scaling of the phase term of Hermite or Laguerre modal polynomial as programmed onto the SLM; secondly, the aperture of the Gaussian mode as defined by the detection fibre and imaged to the plane of the SLM. It is the ratio of these two length scales in the plane of the SLM that defines the detail of the modal measurements and ultimately the degree of orthogonality between the measured modes.

In a classical communication system, one can analyse its information capacity by considering the correlations between the various input and output states; in our case these states are spatial modes. A matrix of probabilities can than be assambled, $P_{i, j}$, for the transmission of mode $i$ and detection of mode $j$. We can then express this matrix in terms of predicted conditional probabilities for a measurement of mode $i$ given a transmission of mode $j$, of $P_{i \mid j}$. The information capacity associated with using these modes in a communication or processing system can then be quantified by a calculation of the mutual information given by [22]

$$
\text { Bits per photon }=\sum_{i, j} P_{i \mid j} \log _{2}\left(\frac{P_{i \mid j}}{P_{i} P_{j}}\right) \text {. }
$$

In a quantum system based upon parametric down-conversion, rather than having a transmitted and detected mode, two detected modes are present instead, measuring the spatial mode of the signal and idler photon respectively. The matrix of probabilities describes now the joint detection of mode $i$ and mode $j$. Similarly the conditional probability is for the detection of mode $i$ given the detection of mode $j$. 


\subsection{Modelling}

In the analysis and subsequent experiment that follows it is assumed that the measurement of spatial modes is undertaken using an SLM to impart the transverse phase structure on the fundamental mode of an optical fibre. This approach is widely used in both classical and quantum experimental systems $[1,2,7,23]$.

Assuming the use of a phase-only structuring of the Gaussian intensity profile, the information capacity, based upon the matrix of probability $P_{i, j}$ is then calculated for a range of lateral scaling, with the scale of the mode expressed as a ratio of the size of the mode to that of the measurement fibre. Figure 1 shows a range of correlation matrices for various ratios, calculated for 10 modes covering all modes of mode order $\mathrm{N} \leq 3$. These predicted correlation strengths were calculated from the overlap integral between the two modes, which were themselves calculated as the Gaussian fibre mode modified by the phase profile imparted by the SLMs. The correlation strengths between all the possible modes can be calculated for different ratios between the size of the mode specified by the SLM and the size of the fibre mode thereby allowing the total mutual information to be calculated as a function of this ratio. Figure 1 also shows the relationship between the ratio of length scales and the mutual information carried by each photon for both LG and HG modal sets.

It is important to note that for all aperture ratios the information capacity of the LG modes equals, and more often exceeds that of the HG modes. This advantage of the LG modal set is readily understood since all modes of different $l$ remain orthogonal to each other irrespective of the size of the circular, on-axis, aperture, i.e. the helicity of the phasefronts is unchanged by the aperture. Therefore it follows that irrespective of the aperture ratio, for an LG modal set, the minimum number of orthogonal modes is $2 \mathrm{~N}+1$, which represents a limiting value for a system with a non optimal aperture ratio. However, the strengths of the correlations between highly correlated modes still depends upon the modes itself (e.g. the strongest correlation is between $l=0, p=0$ and $l=0, p=0$ ). This non uniformity between correlations reduces slightly the mutual information below that which might have been expected from the number of orthogonal modes alone.

By contrast, when dealing with HG modes with a non-optimal aperture ratio, the number of orthogonal modes should not exceed 4. This is limit arises from the symmetry of the modes with can only be either symmetric (EVEN) or antisymmetric (ODD) in the vertical and horizontal axis. Specifically, the symmetry of the modes can be EVEN-EVEN, ODD-ODD, ODD-EVEN or EVEN-ODD, i.e. in the case of restricted aperture, there are only four orthogonal states which limits the information capacity accordingly.

It is important to consider when restricting measurement to a phase only structuring of the detected modes that the LG and HG modal set are not themselves quite optimal. As has been noted previously one can modify slightly the nodal positions within the modes to maximise their orthogonality, even used in conjunction with a measurement fibre of fixed size [23]. For both the modified HG and modified LG modes, the lateral and radial discontinuities in the diffractive element design can be perturbed such that a completely orthogonal modal set can be obtained for a specific lateral scaling with respect to the defining fibre. However, for our specific case of $\mathrm{N} \leq 3$ the optimal aperture close approaches the maximum of $\sum_{n=0}^{N}(n+1)$ even without this optimisation step (especially for the LG set). Note that the maximal bits per photon doesn't peak where the ratio between mode waist as specified on the SLM and fibre equals one. This is due to the fact that for a given size of fibre mode the beam waist of the best fit Laguerre-Gaussian mode is a complicated function of both $l$ and $p$. In general the waist of the resulting LG mode is smaller than that of the fibre mode, hence why the maximum mutual information occurs for ratios $<1$. 

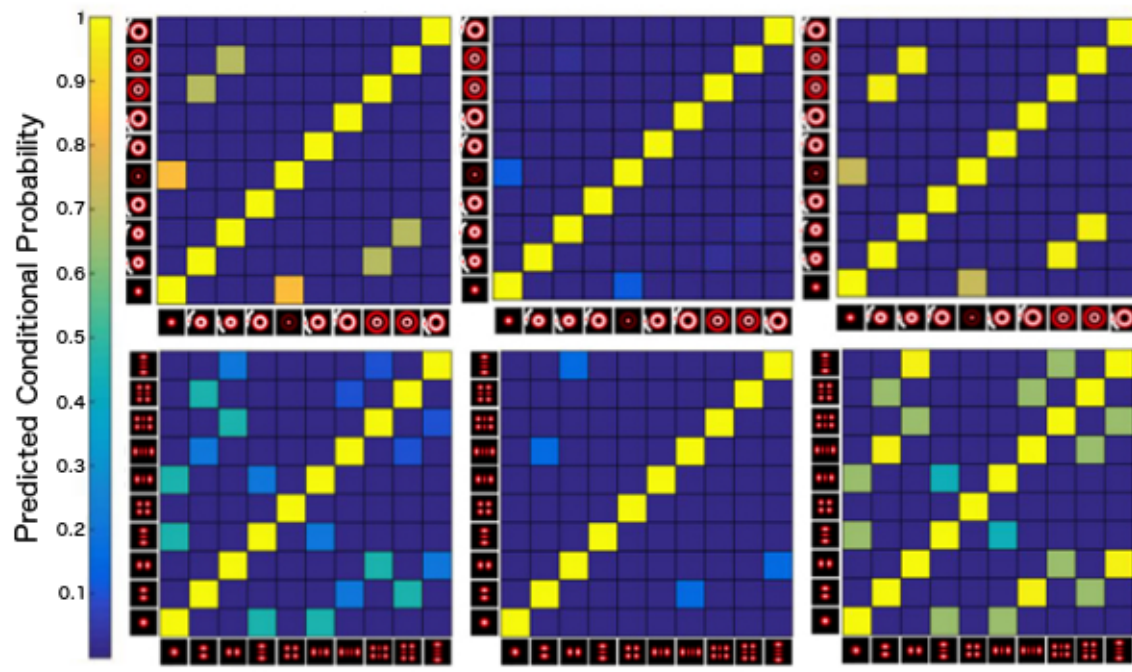

LG

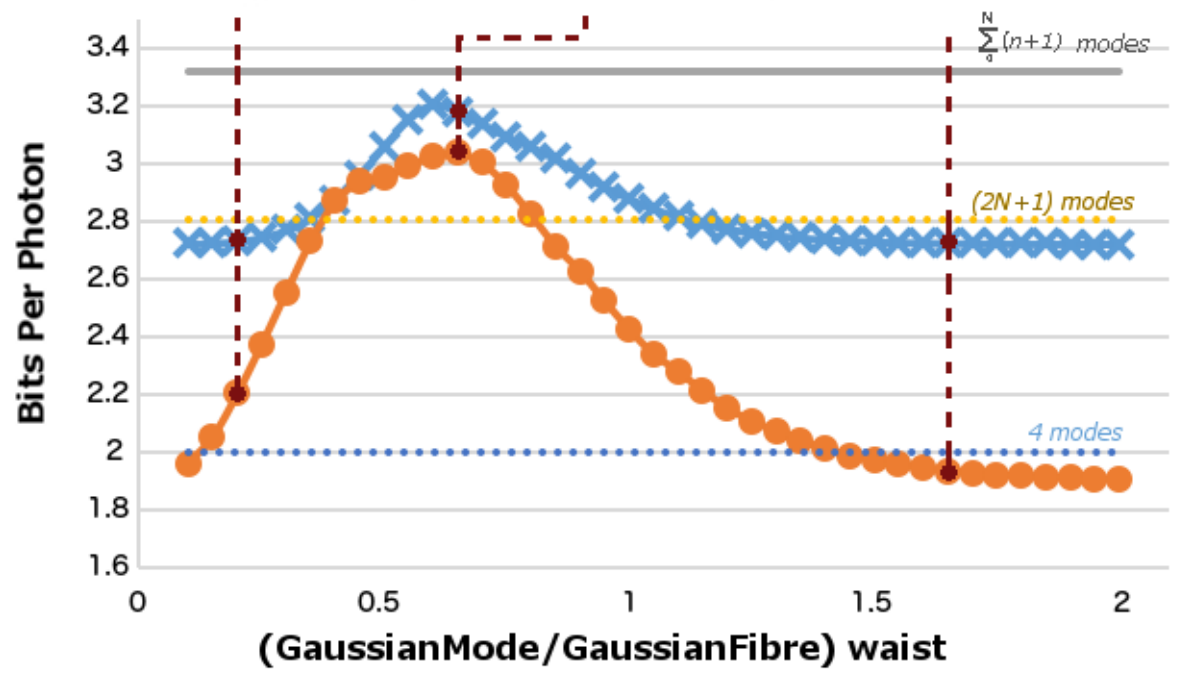

Fig. 1. Data from modelling. The graph shows the value of bits per photon (bpp) calculated for different correlation matrixes of both LG and HG for modes of $\mathrm{N} \leq 3$ and some of the corresponding correlation matrixes. It also shows the value of bpp expected, if the two spatial modes where perfectly correlated $\left(\sum_{n=0}^{N}(n+1)\right)$. 


\subsection{Experiment}

Our experimental system (Fig. 2) comprises of a CW laser at $355 \mathrm{~nm}$ pumping a $3 \mathrm{~mm}$-long crystal of BBO to obtain down-converted signal and idler fields at $710 \mathrm{~nm}$. A longpass filter immediately after the crystal blocks the pump beam and transmits only the $710 \mathrm{~nm}$ signal and idler photons. The signal and the idler beams are separated on a beam splitter and the plane of the crystal is imaged, by the use of the lenses $\mathrm{f} 1(\mathrm{f}=200 \mathrm{~mm})$ and $\mathrm{f} 2(\mathrm{f}=400 \mathrm{~mm})$, onto two separate SLMs. The planes of the SLMs are themselves imaged, by use of the lenses $\mathrm{f} 3(\mathrm{f}=600 \mathrm{~mm})$ and $\mathrm{f} 4(\mathrm{f}=2 \mathrm{~mm})$, to the entrance facets of single mode fibres that are connected to single photon detectors. The outputs of the photon detectors are then connected to a coincidence counter and the coincidence rate is recorded as a function of the measurement states specified by the SLM. In this set up the effective waist of the near-Gaussian fibre-mode in the plane of the SLM is a $2 \mathrm{~mm}$ diameter.

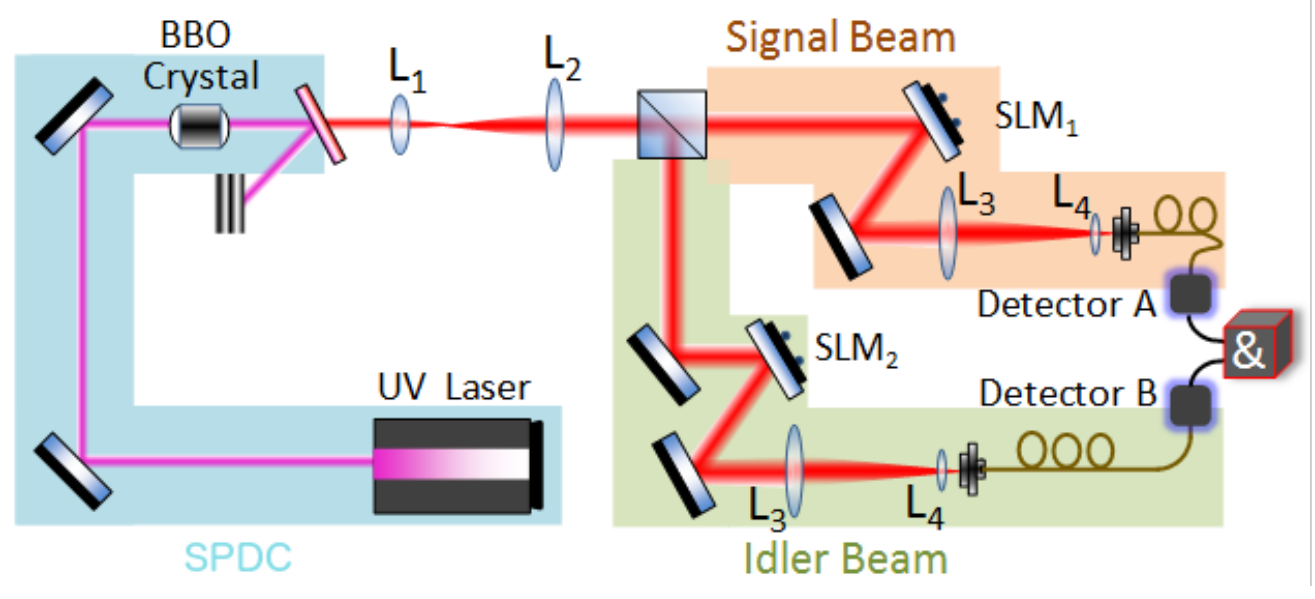

Fig. 2. Experimental set-up. A $355 \mathrm{~nm}$ is used to pump a BBO crystal producing spatially correlated photon pairs. The plane of the crystal is imaged onto two separate SLMs that are themselves imaged onto the entrance facets of single-mode fibres. These fibres are connected to single-photon counters allowing the coincidence count rate to be measured as a function of signal and idler modes.

The physics of parametric down-conversion means that despite the pump beam being a single transverse mode, the down-converted light is emitted into many spatial modes [24]. However, as now demonstrated by numerous groups worldwide, momentum conservation within the down-conversion process means that whenever the SLMs are programmed to detect modes that are complex conjugate of each other, the coincident count rate between the two single-photon detectors is high [9,25-27]. In our laboratories, such configurations have been used to show EPR-like correlations for orbital angular momentum - angle states [4] and violations of Bell-like inequalities [28].

In these present experiments the strength of the correlations between signal and idler spatial modes is measured as defined in either the Hermite-Gaussian or Laguerre-Gaussian modal sets following our numerical analysis above. The result is a measured correlation matrix with each element giving the coincident count rate, $M C_{i, j}$, for the joint detection of modes $i$ and $j$ (Fig. 3). These matrices differs from those simulated especially for elements with low-count rate where accidental coincidences arising within the gate time, $\Delta t$, of counting electronics give weak correlation. Complications can also occur at small measurment waists where SLM 


\section{Optics EXPRESS}

pixilation becomes significant as seen in previous experiments [23]. Given a count rate on the two individual detectors of $S_{i}$ and $S_{j}$ the accidental coincidence rate is estimated as $S_{i} \times S_{j} \times \Delta t$ allowing us to define a quantum contrast expressing the strength of the correlation as a ratio to that expected from classical events [29],

$$
Q C_{i, j}=M C_{i, j} /\left(S_{i} \times S_{j} \times \Delta t\right)
$$
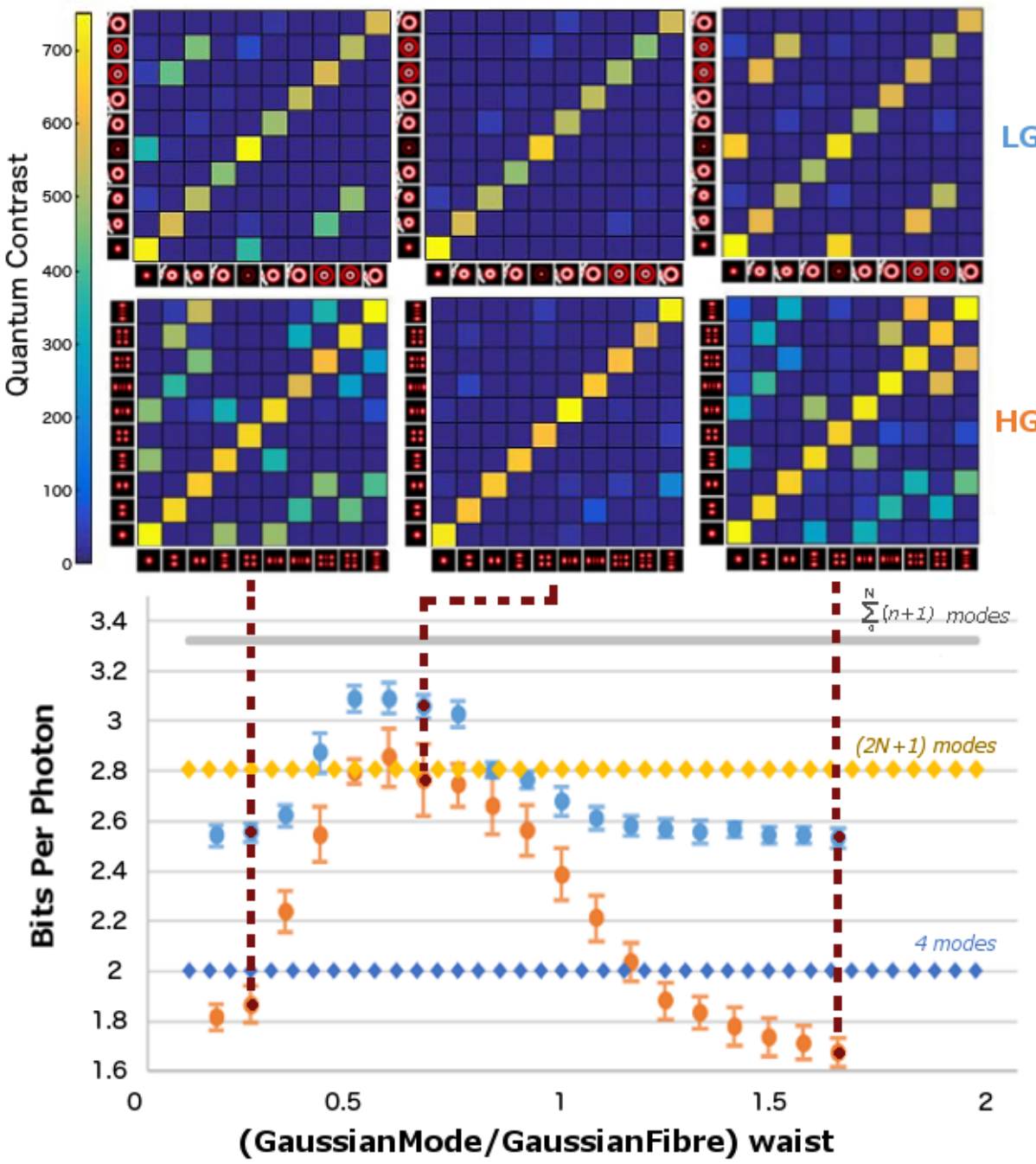

Fig. 3. Experimental Data. The graph shows the value of bits per photon (bpp) calculated for different correlation matrixes of both $\mathrm{LG}$ and $\mathrm{HG}$ for modes of $\mathrm{N} \leq 3$ and some of the corresponding correlation matrixes. It also shows the value of bpp expected, if the two spatial modes where perfectly correlated $\left(\sum_{n=0}^{N}(n+1)\right)$. The raw data used to produce this figure is available in a data archive (Dataset 1) [30].

The visual agreement between these measured and simulated correlation matrices is striking. However, closer inspection reveals that the zeros in the measured matrices are not perfectly zero. 
These non zeros arise from either a modal impurity of the pump leading to a weakening of the correlation, or slight cross-talk in our modal measurements. Either of these issues would impact upon the deployment of a system, but in this work we are interested in the properties of the modes. Therefore in order to illustrate the underlying behaviours of the modes themselves, a background subtraction of the noise from these matrices was made prior to a calculation of their information capacity. This background noise was assessed by taking mid range value of all the matrix elements that appeared in the simulation as zero and subtracting this value from all the measurements within each matrix.

The experiment was then performed for each aperture ratio 10 consecutive times calculating for each run the corresponding bits per photon (bpp). The graph showing the experimental results for the bop as a function of the waist ratios was then calculated from that data by plotting the average of the 10 runs and using the standard deviation of the data to calculate the error bars (see Fig. 3).

It is important to note that the features and trends that our modelling predicted can also be seen in the experimental result. The information capacity of the LG and HG experimental measurements are slightly below those predicted which can be attribute to the difficulties in making high contrast modal measurements.

\section{Discussion}

In this work it has been shown that for the case of restricted transmission and or measurement aperture that information capacity of the LG modal set may exceed that of the HG set. In comparing the simulations with the data analysis it is important to note the importance of QC. In our simulations it is sufficient to calculate the correlation matrix and resulting mutual information based on the strengths of the predicted correlations alone. In our experiments there is a need to account for the dark counts of the detectors, uncorrelated single counts, and the resulting background of accidental correlations, hence in comparing our results to those modelled we use quantum contrast which compensates for this background effect.

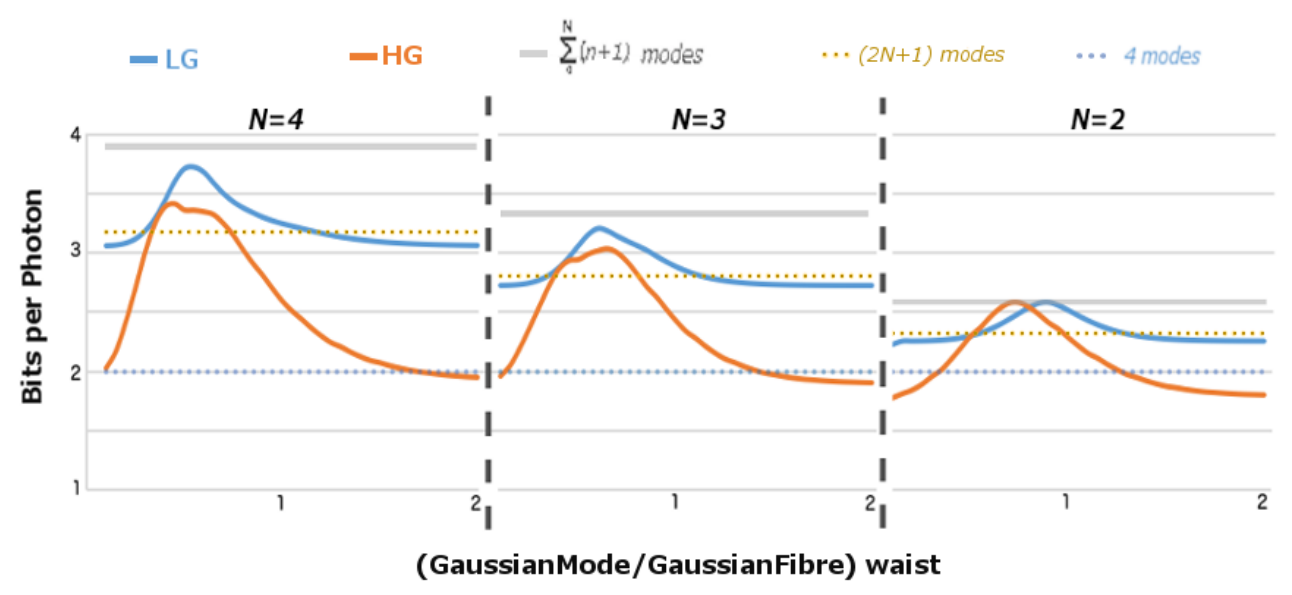

Fig. 4. Comparison of the calculation of the bpp carried by LG and HG modes for $\mathrm{N}=2,3$ and 4 .

Also, for our experimental results, we restricted ourselves to the representative case of a modal set of 10 modes, i.e. modes of order $\mathrm{N} \leq 3$. However, the principle extends to other mode orders, see for example Fig. 4 comparing the bpp for $\mathrm{N}=2,3$ and 4.

Although these results consistently show a superiority of the LG over the HG measurement set, 


\section{Optics EXPRESS}

this is only calculated for the specific case of all the apertures being co-axial and the advantage of the LG set does not necessarily extend to an axis displacement.

Another factor not considered here is that although the LG set may maintain their orthogonality better than the HG, the losses associated with the two sets are not equivalent. Pure LG modes exhibit zero on-axis intensity: hence, a restricted aperture may introduce additional loss compared to the HG, which in a signal-staved environment will further reduce the information capacity. The extent of this loss, and the significance of this on the bpp, depend upon the intensity structuring of the modes and therefore, to the precise measurement plane and should be considered in full within any experimental embodiment of a system.

\section{Conclusions}

We have presented an example where not all modal set are equivalent, and the optimum choice of set depends critically upon the boundary conditions of the experiment and the requirements of the free-space optical link. Considerations of the information capacity of different modal sets applies both to classical and quantum systems. Here, we chose to use a correlated single photon system and coincident measurement to illustrate the effect of modal choice, thereby stressing the relevance of this present work to the recent work of others. However, it is important to realise that our results and conclusions apply to the classical systems too where the two modes are the transmitted and received modes in a communication link.

\section{Funding}

European Union's Seventh Framework Programme (TWISTS); UK Quantum Technology Hub in Quantum Enhanced Imaging (EP/M01326X/1).

\section{Acknowledgments}

M. J. P. acknowledgments the Wolfson foundation and the Royal Society. 